\title{
The downregulation of c-Myc and its target gene hTERT is associated with the antiproliferative effects of baicalin on HL-60 cells
}

\author{
XIA REN $^{1 *}$, ZHIYONG ZHANG $^{1 *}$, JING TIAN $^{2}$, HENGXIAO WANG $^{1}$, GUANHUA SONG $^{1}$, QIANG GUO $^{1}$, \\ JING TIAN $^{1}$, YANG HAN ${ }^{1}$, QIONG LIAO ${ }^{1}$, GUOQIANG LIU $^{3}$, HUIFANG DING ${ }^{3}$ and GUOSHENG JIANG ${ }^{1}$ \\ ${ }^{1}$ Key Laboratory for Rare and Uncommon Diseases of Shandong and Key Laboratory for Tumor Immunology \\ and Chinese Medicine Immunology of Shandong, Institute of Basic Medicine, Shandong Academy of Medical Sciences, \\ Jinan, Shandong 250062; ${ }^{2}$ Laboratory Department, People's Hospital of Zhangqiu, Zhangqiu, Shandong 250200; \\ ${ }^{3}$ Department of Hematology, Shengli Oilfield Central Hospital, Dongying, Shandong 257034, P.R. China
}

Received April 26, 2016; Accepted June 29, 2017

DOI: $10.3892 / \mathrm{ol} .2017 .7039$

\begin{abstract}
Baicalin is a flavonoid compound isolated from Scutellaria baicalensis, a Chinese traditional medicinal herb, and is used as an anti-inflammatory, antibacterial, anxiolytic and hepatoprotective drug. Accumulating evidence has demonstrated that baicalin exhibits potent antitumor properties by suppressing cell growth, arresting cell cycle progression and inducing differentiation or apoptosis in leukemia cell lines. However, whether or not the extrinsic pathway is involved in baicalin-induced apoptosis of leukemia cells and the mechanisms underlying the antitumor activity of baicalin remain unclear. In the present study, the effect of baicalin on the expression of caspase-8, Fas cell surface death receptor (Fas) and Fas ligand in HL-60 cells was assessed, and it was demonstrated that the Fas-mediated extrinsic pathway was also involved in baicalin-triggered cell apoptosis, in addition to the intrinsic pathway. Furthermore, baicalin was able to inhibit the proliferation of HL-60 cells by arresting the cell cycle at the $\mathrm{G}_{0} / \mathrm{G}_{1}$ phase, and by down-regulating Myc proto-oncogene
\end{abstract}

Correspondence to: Dr Huifang Ding, Department of Hematology, Shengli Oilfield Central Hospital, 31 Jinan Road, Dongying, Shandong 257034, P.R. China

E-mail: dingmx2008@126.com

Dr Guosheng Jiang, Key Laboratory for Rare and Uncommon Diseases of Shandong Province and Key Laboratory for Tumor Immunology and Chinese Medicine Immunology of Shandong, Institute of Basic Medicine, Shandong Academy of Medical Sciences, 18877 Jingshi Road, Jinan, Shandong 250062, P.R. China E-mail: jianggsh@hotmail.com

*Contributed equally

Key words: baicalin, HL-60 cells, apoptosis, proliferation, caspases, extrinsic pathway, Myc proto-oncogene protein, human telomerase reverse transcriptase protein (c-Myc) along with its target gene, human telomerase reverse transcriptase. In summary, the results of the present study demonstrated that baicalin was able to inhibit the growth of HL-60 cells through blockade of the $\mathrm{G}_{0} / \mathrm{G}_{1}$ phase of the cell cycle, and significantly induce the apoptosis of cells by activating the intrinsic and extrinsic pathways. The inhibition of HL-60 cell growth was also demonstrated to be mediated by telomerase inhibition through suppression of c-Myc. The results of the present study highlight the possibility of baicalin as a promising regimen for the treatment of AML.

\section{Introduction}

Baicalin is a flavonoid compound isolated from Scutellaria baicalensis (Fig. 1A), a Chinese traditional medicinal herb used as an anti-inflammatory (1), antibacterial, anxiolytic and hepatoprotective drug (2). Accumulating evidence has demonstrated that baicalin exhibits potent antitumor properties by suppressing cell growth, arresting the cell cycle and inducing differentiation or apoptosis in leukemia cell lines $(3,4)$, without affecting primary, or normal cells (5), which raises the possibility of using baicalin to treat patients with leukemia. Baicalin has been documented to induce apoptosis of the Jurkat T-cell acute lymphoblastic leukemia (T-ALL) cell line via the mitochondrial pathway, which involves marginal generation of intracellular reactive oxygen species (ROS), an increase in the cytosolic fractions of cytochrome $c$ and disruption of the mitochondrial transmembrane potential $(\Delta \Psi \mathrm{m})(6)$. Another study revealed that baicalin exhibited a remarkable cytotoxic effect in T-ALL cell line CCRF-CEM, and triggered apoptosis regulator Bcl-2 (Bcl-2)-dependent, but not p53-dependent cell apoptosis (7).In addition, cyclin-dependent kinase inhibitor p27 has been suggested to serve a role in baicalin-induced apoptosis and cell cycle arrest (3). A study by Lu et al (8) confirmed that baicalin induced apoptosis in human promyelocytic leukemia HL-60 cells through the mitochondrial-involved pathway and endoplasmic reticulum-induced apoptotic cell death. While to date, whether the extrinsic pathway (death receptor pathway) is involved in apoptosis induced by baicalin in leukemia cells 
has not been clarified, and the mechanisms underlying the antitumor activity of baicalin remain unclear.

The proliferation of HL-60 cells has also been demonstrated to be inhibited by baicalin (8); however, its underlying mechanism requires further elucidation. Myc proto-oncogene protein (c-Myc) is a transcription factor that participates in various cellular functions, including cell-cycle progression, proliferation, apoptosis and terminal differentiation (9). The overexpression of c-Myc mRNA and its encoded protein has been associated with neoplastic transformation in a variety of tumors (10). Therefore, perturbation of c-Myc levels is essential for influencing the growth of malignant cells, particularly those of hematopoietic origin (11). Wogonin, another major bioactive flavonoid extracted from Scutellaria baicalensis has been revealed to inhibit HL-60 cell growth via telomerase inhibition through suppression of c-Myc (12). Due to the similar molecular structure between baicalin and wogonin (Fig. 1B), it was hypothesized that baicalin may be able to inhibit the growth of HL-60 cells via the same mechanism as wogonin.

In the present study, the effect of baicalin on the expression of caspase-8, Fas cell surface death receptor (Fas), Fas ligand (FasL), death receptor (DR)4 and DR5 in HL-60 cells was assessed, and it was identified that the Fas-mediated extrinsic pathway was involved in baicalin-triggered cell apoptosis, in addition to the intrinsic pathway. Furthermore, baicalin was able to inhibit the proliferation of HL-60 cells by arresting cells at the $\mathrm{G}_{0} / \mathrm{G}_{1}$ phase and downregulating c-Myc along with its target gene, human telomerase reverse transcriptase (hTERT).

\section{Materials and methods}

Reagents. Baicalin was provided by Professor Xiao Wang (Shandong Analysis and Test Center, Shandong Academy of Sciences, Jinan, China). Baicalin was dissolved in DMSO at a stock concentration of $10 \mathrm{mg} / \mathrm{ml}$ and stored at $-20^{\circ} \mathrm{C}$. Hoechst 33342 was obtained from Beyotime Institute of Biotechnology (Nantong, China). $\beta$-actin (cat. no. sc130065; dilution, 1:500) and c-Myc (cat. no. sc-47694; dilution, 1:400) antibodies were purchased from Santa Cruz Biotechnology, Inc. (Dallas, TX, USA). Antibodies directed against apoptosis regulator $\mathrm{Bax}$ (Bax; cat. no. 2774; dilution, 1:1,000), Bcl-2 (cat. no. 2872; dilution, 1:1,000), cleaved caspase-3 (cat. no. 9661; dilution, 1:1,000), caspase-9 (cat. no. 9502; dilution, 1:1,000), caspase-8 (cat. no. 9746; dilution, 1:1,000), Fas (cat. no. 4233; dilution, 1:1,000) and Fas ligand (FasL; cat. no. 4273; dilution, 1:1,000) were purchased from Cell Signaling Technology, Inc. (Danvers, MA, USA). Horseradish peroxidase (HRP)-conjugated immunoglobulin $\mathrm{G}$ anti-mouse (cat. no. SA00001-1; dilution, 1:10,000) and anti-rabbit (cat. no. SA00001-2; dilution, 1:10,000) antibodies were purchased from ProteinTech Group, Inc. (Chicago, IL, USA). Cell Counting Kit-8 (CCK-8) was purchased from Dojindo Molecular Technologies, Inc. (Kumamoto, Japan).

Cell culture and proliferation assay. HL-60 cells were cultured at Key Laboratory for Tumor Immunology and Chinese Medicine Immunology of Shandong Province (Institute of Basic Medicine, Shandong Academy of Medical Sciences, Jinan, China). The cells were cultured in RPMI-1640 (Gibco; Thermo Fisher Scientific, Inc., Waltham, MA, USA)

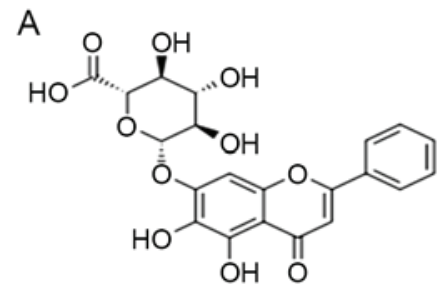

B

Figure 1. Chemical structure of baicalin and wogonin. Chemical structure of (A) baicalin and (B) wogonin

supplemented with $10 \%$ newborn calf serum (Gibco; Thermo Fisher Scientific, Inc.), $100 \mathrm{IU} / \mathrm{ml}$ penicillin and $100 \mathrm{IU} / \mathrm{ml}$ streptomycin in a $5 \% \mathrm{CO}_{2}$ atmosphere at $37^{\circ} \mathrm{C}$. Logarithmically growing HL-60 cells were seeded on a 96-well plate at a density of $1 \times 10^{4}$ cells/well in $50 \mu \mathrm{l}$ of medium in triplicate, and then different concentrations of baicalin $(5,10,20,40$ and $80 \mu \mathrm{g} / \mathrm{ml})$ in $50 \mu \mathrm{l}$ were added. Cells treated with $0.1 \%(\mathrm{v} / \mathrm{v})$ DMSO were used as the control. After incubation for $24 \mathrm{~h}$, cell proliferation was detected using CCK-8, according to the manufacturer's protocol as previously described (13).

Cell cycle distribution. Following baicalin $(20 \mu \mathrm{g} / \mathrm{ml})$ treatment for different durations, cells $\left(3 \times 10^{5}\right)$ were washed twice with ice-cold PBS, and fixed in cold $75 \%$ ethanol at $4{ }^{\circ} \mathrm{C}$ for at least $24 \mathrm{~h}$. Then, the cells were rinsed with PBS, and resuspended using $0.5 \mathrm{mg} / \mathrm{ml} \mathrm{RNaseA}$ and $20 \mu \mathrm{g} / \mathrm{ml}$ propidium iodide (PI) prior to incubation for $20 \mathrm{~min}$ at room temperature in the dark. Subsequently, the cells were analyzed using an Elite-ESP flow cytometer (Beckman Coulter, Inc., Brea, CA, USA).

Analysis of apoptosis. Hoechst 33342 staining was used to visualize the change in nuclear morphology. Following baicalin $(20 \mu \mathrm{g} / \mathrm{ml})$ treatment for $24 \mathrm{~h}$, cells were washed twice with PBS and fixed with $4 \%$ paraformaldehyde for $15 \mathrm{~min}$ at room temperature. Then, washed cells were stained with $1 \mu \mathrm{g} / \mathrm{ml}$ Hoechst 33342 for $15 \mathrm{~min}$ at room temperature in the dark. Cells were observed under a fluorescence microscope and imaged. Apoptotic cells were characterized by condensed or fragmented chromatin, while normal nuclei were smooth, symmetric and oval. To quantify the cells undergoing apoptosis, Annexin V/PI (BD Pharmingen; BD Biosciences, San Jose, CA, USA) double staining was performed according to the manufacturer's protocol. Briefly, the cells were harvested by centrifugation for $10 \mathrm{~min}$ at $800 \mathrm{x} g$ at $4^{\circ} \mathrm{C}$. Subsequently, the cells were washed twice with ice-cold PBS and re-suspended in $500 \mu \mathrm{l}$ of $1 \mathrm{X}$ binding buffer, stained with Annexin V and PI for $15 \mathrm{~min}$ at room temperature. Apoptotic cells appeared as Annexin V-positive and PI-positive.

Assays for analysis of $\Delta \Psi m$. Fluorescent dye rhodamine 123 staining and flow cytometric analysis were performed to assess the $\Delta \Psi \mathrm{m}$. Briefly, following baicalin treatment for $24 \mathrm{~h}$, the cells were incubated with $10 \mathrm{mg} / \mathrm{ml} \mathrm{Rh123}$ for $30 \mathrm{~min}$ at $37^{\circ} \mathrm{C}$, and washed twice with PBS, then analyzed using fluorescence-activated cell sorting.

Reverse transcription-semi-quantitative polymerase chain reaction ( $R T$-sqPCR) assays. Total RNA was extracted from 
HL-60 cells that had been cultured with baicalin $(20 \mu \mathrm{g} / \mathrm{ml})$ for different durations using TRIzol (Invitrogen; Thermo Fisher Scientific, Inc.) according to the manufacturer's protocol. RT-PCR was performed as described previously (13). The primers used in the present study were as follows: Fas (238 bp) forward, 5'-GGACCCAGAATACCAAGTGC-3' and reverse, 5'-GCCACTGTTTCAGGATTTAAGG-3'; hTERT (198 bp) forward, 5'-TCACCTCGAGGGTGAAGGCACT GTT-3' and reverse, 5'-ATGCTGGCGATGACCTCCGTGA-3'; c-Myc (180 bp) forward, 5'-GATTCTCTGCTCTCCTCG AC-3' and reverse, 5'-TCCAGACTCTGACTTTTGC-3'; DR5 (389 bp) forward, 5'-TGCAGCCGTAGTCTTGATTG-3' and reverse, 5'-GCACCAAGTCTGCAAAGTCA-3'; DR4 (307 bp) forward, 5'-CAGAGGGATGGTCAAGGTCAAG-3' and reverse, 5'-TTGCTGCTCAGAGACGAAAGTG-3'; $\beta$-actin (540 bp) forward, 5'-GTGGGGCGCCCCAGGCAGGCA CCA-3' and reverse, 5'-CTCCTTAATGTCACGCACGAT TTC-3'. The following thermocycling conditions were maintained: $94^{\circ} \mathrm{C}$ for $5 \mathrm{~min} ; 30$ cycles of $94^{\circ} \mathrm{C}$ for $45 \mathrm{sec}, 55^{\circ} \mathrm{C}$ for $45 \mathrm{sec}$ and $72^{\circ} \mathrm{C}$ for $1 \mathrm{~min}$; and $72^{\circ} \mathrm{C}$ for $10 \mathrm{~min}$. The PCR products were electrophoresed on $1.5 \%$ agarose gels. The software used to quantify the bands was AlphaImager 2200 (version 0.2.1.0; Alpha Innotech, Tarzana, CA, USA).

Western blot analysis. For immunoblotting, HL-60 cells treated with $20 \mu \mathrm{g} / \mathrm{ml}$ baicalin for 12 and $24 \mathrm{~h}$ were washed twice with cold PBS and lysed using extraction buffer (50 mM Tris- $\mathrm{HCl} \mathrm{pH} 7.4,1 \mathrm{mM}$ phenylmethylsulfonyl fluoride, $150 \mathrm{mM} \mathrm{NaCl}, 1 \mathrm{mM}$ EDTA, $1 \%$ Triton $\mathrm{X}-100,0.5 \%$ deoxycholate, and $0.1 \% \mathrm{SDS}$ ) for $15 \mathrm{~min}$ on ice. Protein concentrations were measured using the Bradford protein assay (Beyotime Institute of Biotechnology). Protein samples $(50 \mu \mathrm{g})$ were electrophoresed using 12\% SDS-PAGE, transferred onto a polyvinylidene difluoride membrane. After blocking with Tris buffered saline-Tween-20 containing 5\% non-fat dry milk for $1 \mathrm{~h}$ at room temperature, the membranes were incubated with primary antibodies against $\beta$-actin, cleaved caspase-3, caspase-8, caspase-9, Fas, FasL, c-Myc, Bcl-2 and Bax overnight at $4^{\circ} \mathrm{C}$. Subsequently, membranes were incubated with HRP-conjugated secondary antibodies for $1 \mathrm{~h}$ at room temperature. The protein bands were visualized using an enhanced chemiluminescence detection system (EMD Millipore, Billerica, MA, USA) and imaged using a LAS-4000 mini luminescent image analyzer (Fujifilm, Tokyo, Japan). The software used to analyze the protein band density was Multi Gauge (version 3.0; Fujifilm, Tokyo, Japan).

Statistical analysis. Results are expressed as the mean \pm standard error mean. The data were analyzed using one-way analysis of variance and Tukey's honest significant test post hoc analysis using SPSS (version 13.0; SPSS, Inc., Chicago, IL, USA). $\mathrm{P}<0.05$ was considered to indicate a statistically significant difference.

\section{Results}

Baicalin inhibits the proliferation of HL-60 cells, and c-Myc together with hTERT participates in its growth arrest. The effect of baicalin on the growth of HL-60 cells was detected using a CCK-8 assay. As presented in Fig. 2A, baicalin inhibited the proliferation of HL60 cells in a dose-dependent manner with an $\mathrm{IC}_{50}$ value of $21.8 \mu \mathrm{g} / \mathrm{ml}$ at $24 \mathrm{~h}$. Therefore, a baicalin concentration of $20 \mu \mathrm{g} / \mathrm{ml}$ was selected to perform the majority of the following experiments. At this concentration, baicalin arrested cell growth in a time-dependent manner (Fig. 2B).

c-Myc possesses an essential function in controlling cell growth. To investigate the roles of c-Myc in growth inhibition, the mRNA and protein levels of c-Myc gene expression were detected in HL-60 cells exposed to $20 \mu \mathrm{g} / \mathrm{ml}$ baicalin for different durations. As presented in Fig. 2C-F, baicalin resulted in a significant downregulation of c-Myc mRNA and protein levels compared with untreated cells. In line with the aforementioned results, it was demonstrated that the mRNA expression of hTERT, the catalytic subunit of the telomerase holoenzyme complex, a direct transcriptional target of c-Myc (14), consequently decreased (Fig. 2C and D).

Baicalin causes $G_{0} / G_{1}$ arrest in $H L-60$ cells. To investigate the mechanisms underlying growth inhibition by baicalin in HL-60 cells, cell cycle analysis was then performed using flow cytometry. As presented in Fig. 3, the time-course study revealed that exposure of HL-60 cells to baicalin significantly increased the proportion of cells in $\mathrm{G}_{0} / \mathrm{G}_{1}$ phase from $(29.2 \pm 0.5 \%)$ in the vehicle treated cells to $(37.6 \pm 3.2 \%$; $P<0.05)$, accompanied by a reduction in the percentages of HL-60 cells in the $G_{2} / M$ and $S$ phases. In addition, cells in the sub- $G_{1}$ phase rapidly increased at $24 \mathrm{~h}$ and the sub- $\mathrm{G}_{1}$ hypodiploid population reached $20.1 \pm 2.9 \%$ (data not shown). The data indicated that inhibition in cell cycle progression and induction of apoptosis may be associated with the inhibitory effect of baicalin treatment on the proliferation of HL-60 cells.

Baicalin induces apoptosis in HL-60 cells. To further characterize apoptosis induced by baicalin, Hoechst 33342 staining and Annexin V/PI analysis was performed. The nuclei of untreated cells were round and large in size, exhibiting homogeneous blue fluorescence. In contrast, a number of the cells treated with baicalin for $24 \mathrm{~h}$ were observed with condensed or fragmented nuclei, which are characteristics of cell apoptosis (Fig. 4A). In addition, the induction of apoptosis was time-dependent (Fig. 4B and C). Baicalin induced apoptosis in $12.6 \pm 4.3 \%$ of HL-60 cells after $12 \mathrm{~h}$ treatment, while it induced $32.3 \pm 5.1 \%$ of cells after $24 \mathrm{~h}$ treatment.

Baicalin induces the activation of caspases. To determine the mechanism of apoptosis induction by baicalin treatment, the activities of caspases, which have been demonstrated to be essential mediators in the apoptosis pathway (15), were analyzed using western blotting. Treatment of cells with baicalin resulted in marked time-dependent caspase-3, caspase- 8 and caspase- 9 activation, and cleavage, which indicated that the extrinsic and intrinsic pathways were associated with apoptosis induced by baicalin (Fig. 5).

Baicalin-induced apoptosis is mediated through intrinsic pathway. The decline in $\Delta \Psi \mathrm{m}$ has been considered to be an important event in intrinsic pathway, which leads to matrix condensation and rapid cytochrome $c$ release (16), 
A

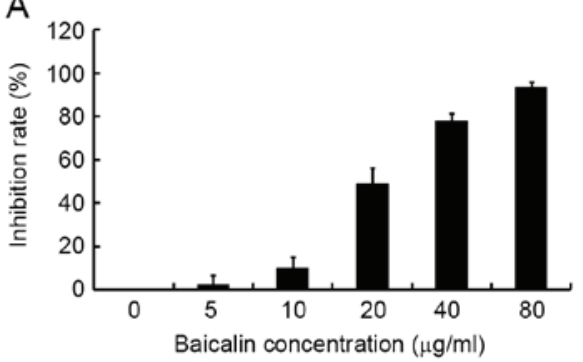

C

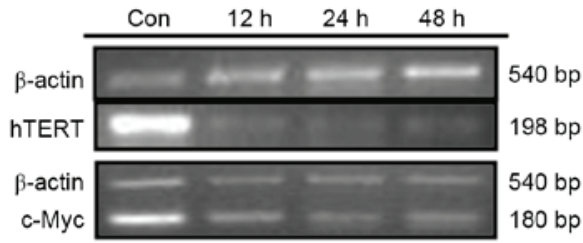

$\mathrm{E}$

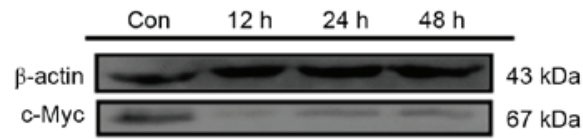

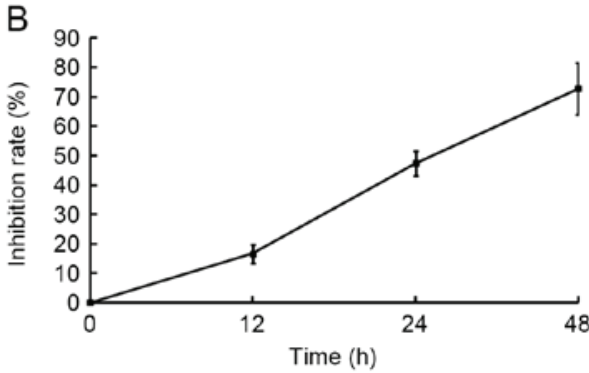
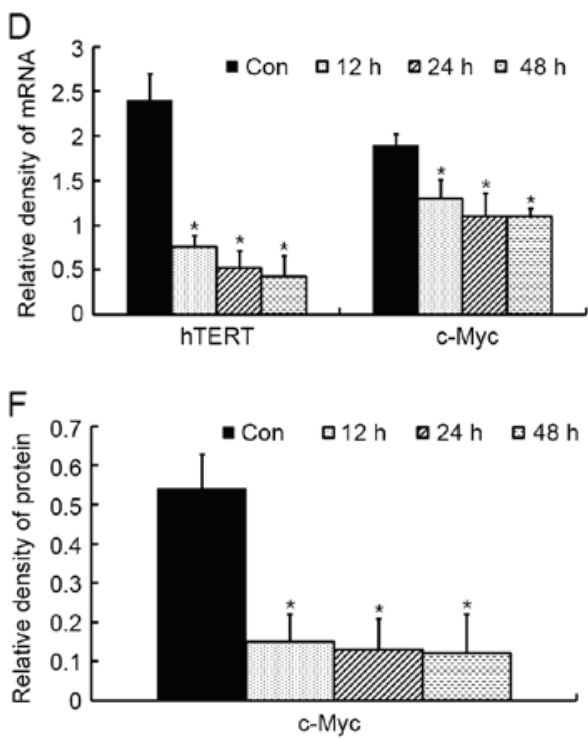

Figure 2. Inhibitory effect of baicalin on the growth of HL-60 cells. HL-60 cells were treated with (A) various concentration of baicalin for $24 \mathrm{~h}$; and were treated with (B) $20 \mu \mathrm{g} / \mathrm{ml}$ baicalin for the indicated time $(0-48 \mathrm{~h})$. The cell viability was determined by using a Cell Counting Kit- 8 assay, the cell growth inhibition rate was calculated as described in the Materials and methods. Values represent the mean \pm standard deviation of experiments performed in triplicate. (C) Baicalin downregulated the expression of c-Myc and hTERT mRNA. (D) The semi-quantitative analysis of c-myc and hTERT mRNA was calculated and is presented. (E) Baicalin downregulated the expression of c-Myc protein. (F) Quantification of c-Myc protein expression in HL- 60 cells. "P<0.01 vs. control. Con, control, hTERT, human telomerase reverse transcriptase; c-Myc, Myc proto-oncogene protein.
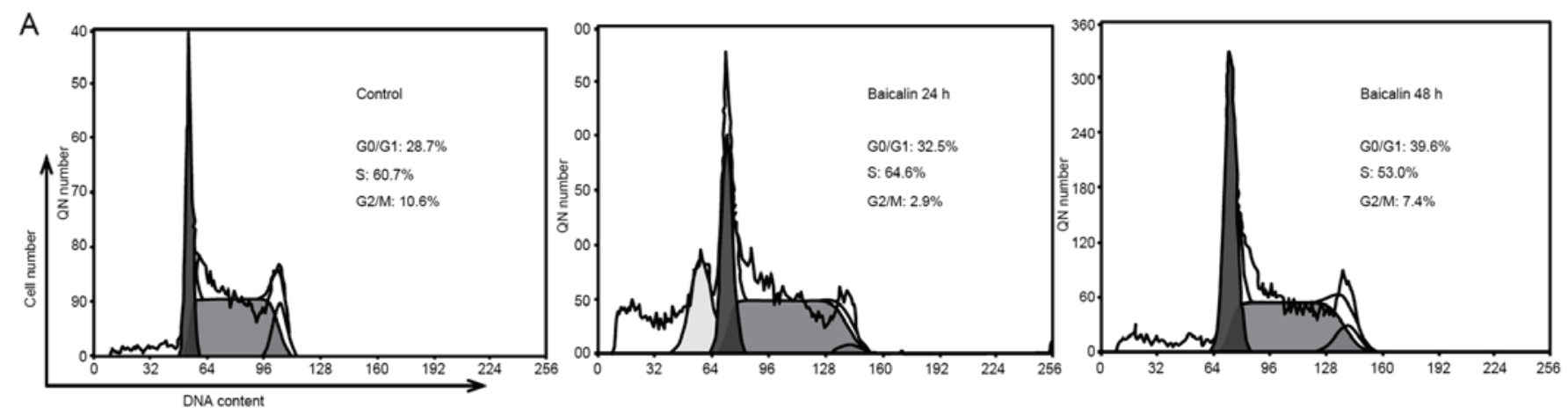

B

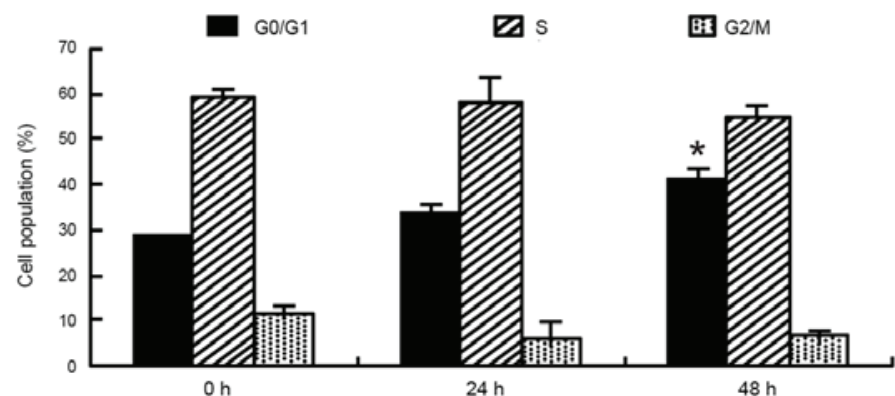

Figure 3. Baicalin induces $\mathrm{G}_{0} / \mathrm{G}_{1}$ cell cycle arrest in HL-60 cells. (A) Following exposure to baicalin $(20 \mu \mathrm{g} / \mathrm{ml})$ for the indicated times (0-48 h), HL-60 cells were stained with propidium iodide, then the DNA content was analyzed through flow cytometry. (B) Quantitative data from A. Values represent the mean \pm standard deviation $(\mathrm{n}=3)$. ${ }^{*} \mathrm{P}<0.05$ vs. control. 
A
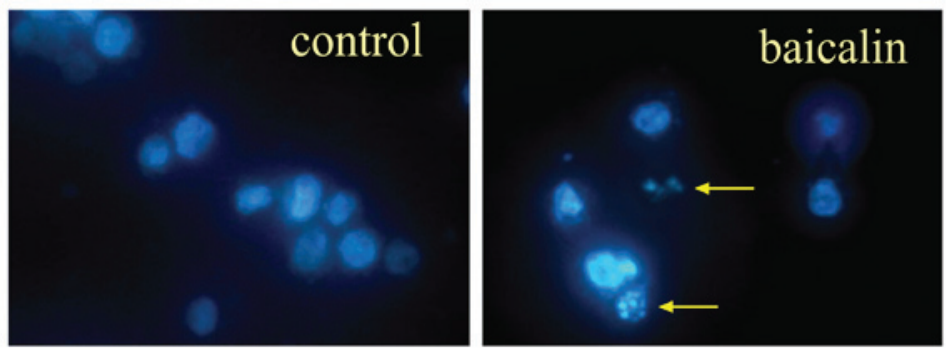

B
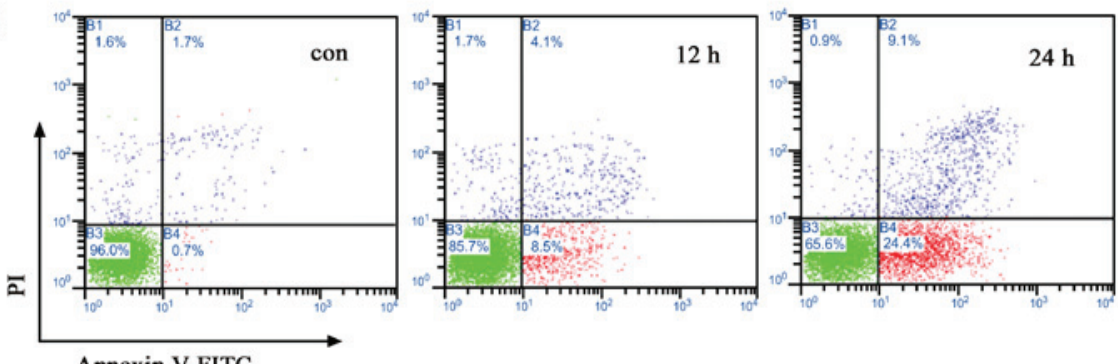

Annexin V-FITC

C

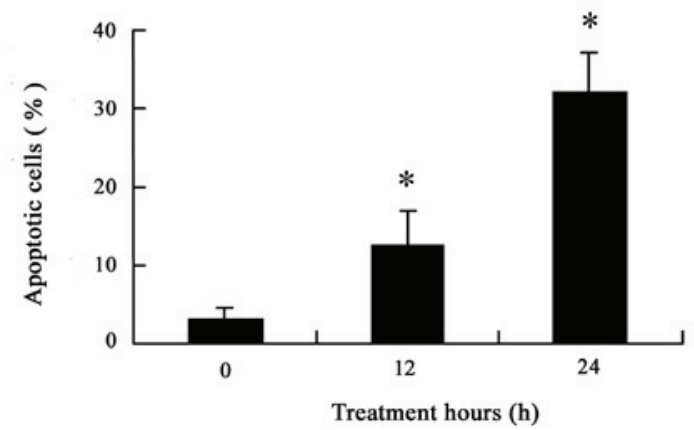

Figure 4. Baicalin induced apoptosis in HL-60 cells. (A) Baicalin induced morphological changes in HL-60 cells. HL-60 cells were treated with or without $20 \mu \mathrm{g} / \mathrm{ml}$ baicalin for $24 \mathrm{~h}$, then fixed and stained with Hoechst 33342. Condensed or fragmented nuclei were considered as apoptotic cells (magnification, x400). Arrows indicate apoptotic cells. (B) HL-60 cells were treated with $20 \mu \mathrm{g} / \mathrm{ml}$ baicalin for the indicated times (0-24 h), stained with Annexin V and PI, and then analyzed using flow cytometry. Cells in the lower right hand quadrant (Annexin $\mathrm{V}^{+} / \mathrm{PI}^{-}$) correspond to early apoptotic cells, whereas cells appearing in the right upper quadrant (Annexin $\mathrm{V}^{+} / \mathrm{PI}^{+}$) correspond to late apoptotic cells. (C) Early and late apoptotic percentages from dot plots were combined to estimate total apoptosis. Values represent the mean \pm standard deviation following experiments performed in triplicate. ${ }^{*} \mathrm{P}<0.01$ vs. control. PI, propidium iodide.

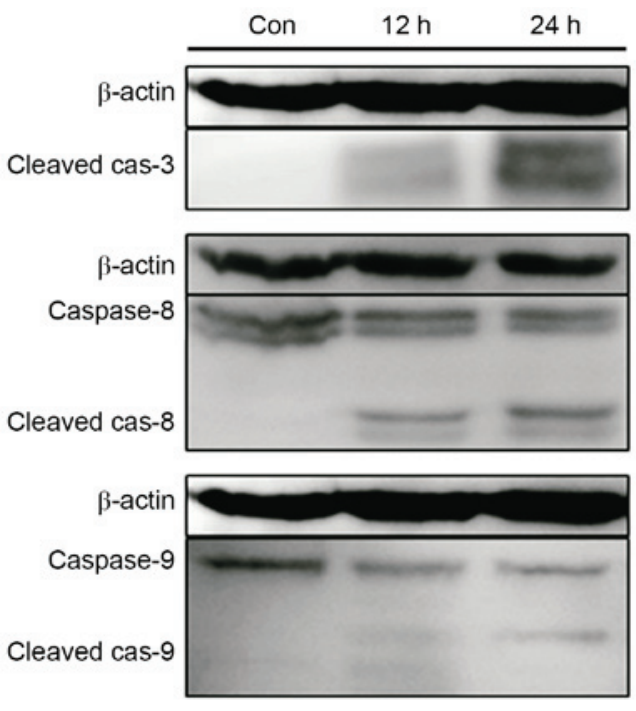

Figure 5. Baicalin treatment of HL-60 cells is associated with increased cleavage of caspase-3, caspase- 8 and caspase-9. HL-60 cells were treated with baicalin for 12 and $24 \mathrm{~h}$, then the cell lysates were subjected to western blot analysis to determine the protein level of caspase-8, caspase-9 and cleaved caspase-3. $\beta$-actin was used as the loading control. All blots were repeated at least three times and one representative blot is presented. Con, control; cas, caspase. followed by caspase- 9 activation. Flow cytometry analysis of Rh123-stained HL-60 cells was performed to investigate the intrinsic pathway. As presented in Fig. 6A, treatment of HL-60 cells with baicalin for $24 \mathrm{~h}$ led to a marked decrease in Rh123 fluorescence compared with the control group, presenting dissipation of $\Delta \Psi \mathrm{m}$.

The Bcl-2 family of proteins is a key factor in the regulation of cytochrome $c$ release from mitochondria (17). To further confirm the involvement of the intrinsic pathway, anti-apoptotic factor Bcl-2 and pro-apoptotic factor Bax were detected using western blotting. The protein expression of Bax was increased notably, but that of Bcl-2 decreased following exposure of HL-60 cells to baicalin for 12 and $24 \mathrm{~h}$ (Fig. 6B). As a result, the ratio of Bcl-2/Bax declined significantly (Fig. 6C). These results demonstrated that the intrinsic signaling pathway was involved in baicalin-treated apoptosis, in accordance with the previous studies $(6,8)$.

Baicalin induces apoptosis through the Fas-mediated extrinsic pathway. To clarify which receptor mediated the extrinsic pathway, the death receptors, including DR4, DR5 and Fas, were detected using RT-PCR. The results revealed that exposure of HL-60 cells to baicalin for $24 \mathrm{~h}$ significantly 
A

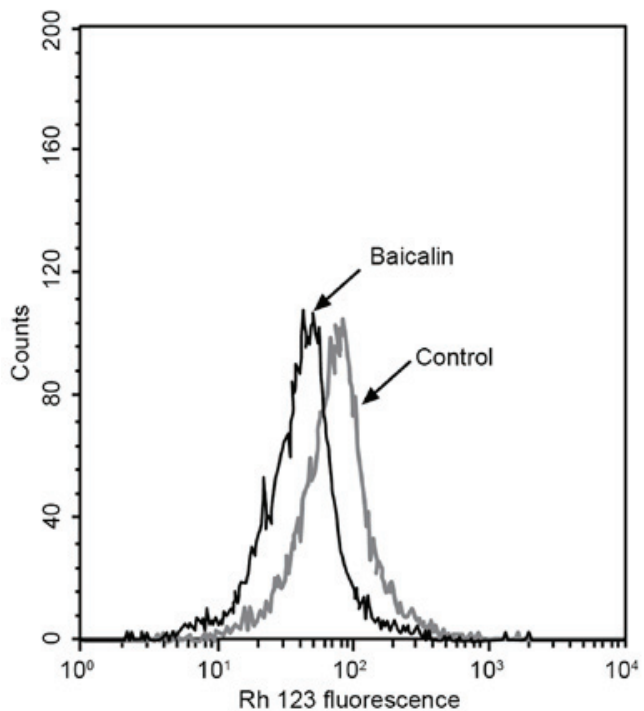

B

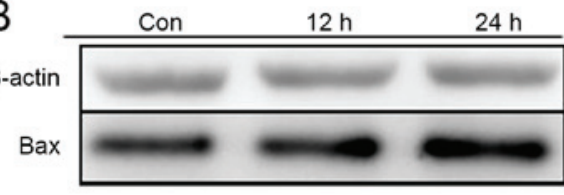

$\beta$-actin

$\mathrm{BCl}-2$

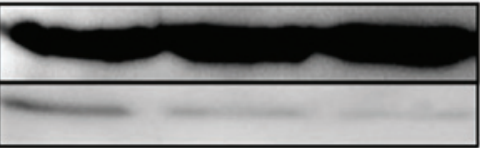

C

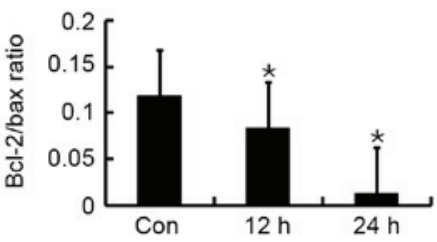

Figure 6. The intrinsic apoptotic pathway is involved in baicalin-induced apoptosis. (A) Changes in the mitochondrial membrane potential in baicalin-treated HL-60 cells as determined through flow cytometry analysis. The fluorescence intensity shifted from a higher to a lower level indicating a decrease in the mitochondrial transmembrane potential. (B) HL-60 cells were treated with baicalin for 12 and $24 \mathrm{~h}$, then the cell lysates were subjected to western blot analysis to determine the protein level of Bax, and Bcl-2. $\beta$-actin was used as the loading control. All blots were repeated at least three times and one representative blot is presented. (C) The histogram represents ratio of values of Bcl-2/Bax. "P<0.05 vs. control. Con, control; Bcl-2, apoptosis regulator Bcl-2; Bax, apoptosis regulator Bax.

A

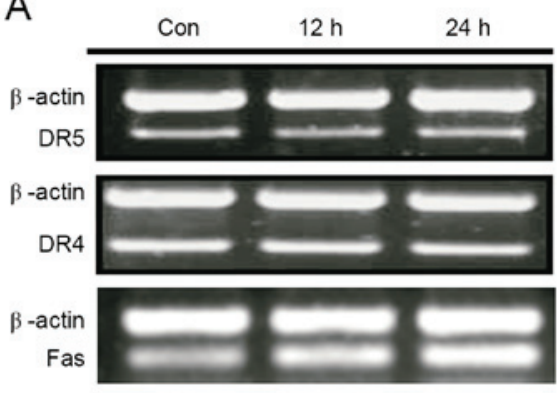

C

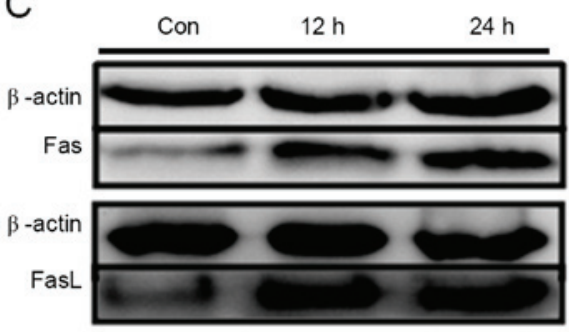

B
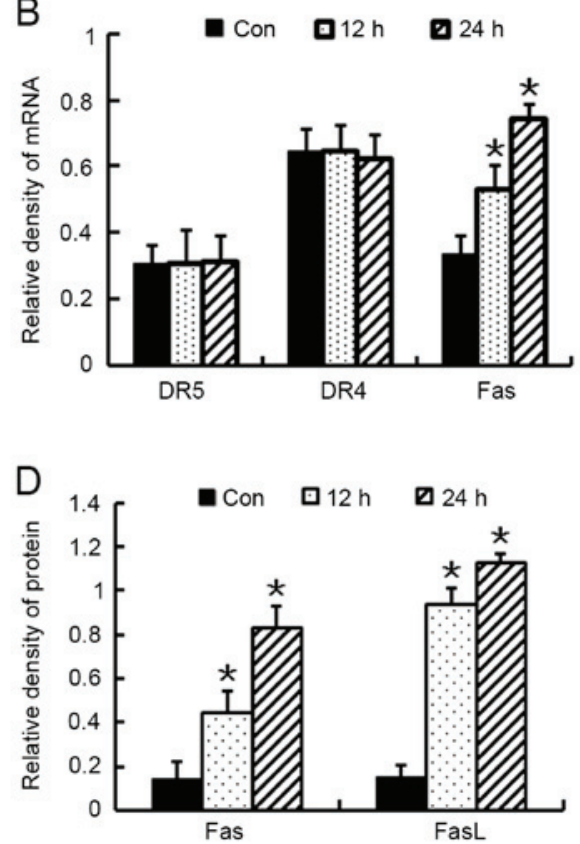

Figure 7. The extrinsic apoptotic pathway is involved in baicalin-induced apoptosis. (A) HL-60 cells were treated with baicalin for 12 and $24 \mathrm{~h}$, then the mRNA expression of DR4, DR5 and Fas were detected using reverse transcription-semi-quantitative polymerase chain reaction. Each amplification was performed at least three times. (B) Semi-quantitative analysis was performed using ImageJ software. (C) HL-60 cells were treated with baicalin for 12 and $24 \mathrm{~h}$, then the protein expression of Fas and FasL were analyzed using western blotting. $\beta$-actin was used as the loading control. Three independent studies yielded equivalent results and representative blots are presented. (D) Quantification of Fas and FasL protein expression in HL-60 cells. "P<0.01 vs. control. Fas, Fas cell surface death receptor; FasL, Fas ligand; DR, death receptor; con, control.

triggered Fas expression, but no significant effects of baicalin on the mRNA levels of DR4 or DR5 were noted (Fig. 7A and B). Western blot assays were then performed, which further confirmed that baicalin induced FasL and Fas expression throughout the time period examined (Fig. 7C and D). The data presented in the present study suggest that activation of the extrinsic Fas-associated pathway partially contributed to baicalin-induced apoptosis. 


\section{Discussion}

As previously reported (8), the present study confirmed that baicalin exhibits high dose- and time-dependent anticancer activity. To determine the molecular mechanisms of the inhibitory effect of baicalin in HL-60 cells, cell cycle analysis was performed using flow cytometry. The results demonstrated that baicalin induced significant $\mathrm{G}_{0} / \mathrm{G}_{1}$ arrest $48 \mathrm{~h}$ later; however, it was reported by Ikezoe et al (3) that baicalin arrested HL-60 cells at the $G_{2} / M$ phase. Consistent with the $G_{0} / G_{1}$ arrest, the results of our previous study demonstrated the baicalin upregulated the expression of cyclin-dependent kinase inhibitors, including p21 and p27 (13), which are involved in the $\mathrm{G}_{1}$ to $\mathrm{S}$ transition (18). It was concluded that baicalin reduced cell viability partially through arresting cells at the $G_{0} / G_{1}$ phase However, the appearance of the hypodiploid peak in the cell cycle distribution demonstrated that apoptosis also contributed to cell proliferation inhibition by baicalin. Furthermore, the cell morphological changes observed and Annexin V/PI staining results further confirmed this, and are concordant with preceding reports $(3,4,6,8)$.

It is well known that caspases, a conserved family of cysteine proteases, serve a central role in the execution of cell apoptosis (19). They are generally divided into two classes: The initiator caspases, which include caspase-2, -8, -9 and -10 ; and the effector caspases, which include caspases-3, -6 and -7 (15). Classical apoptosis occurs through the extrinsic and intrinsic pathway in all mammalian cells. Both pathways converge on activation of initiator and effector caspases (15). The former depends on death receptors, including Fas or TNF-related apoptosis-inducing ligand (TRAIL) receptors, DR4 and DR5 (20). When bound by their specific death ligand, these receptors are activated, resulting in the formation of a death-inducing signaling complex and activation of the initiator caspase-8, which further directly or indirectly activates effector caspase-3 (20). The latter depends on mitochondria. Once the apoptotic stimuli are received, including heat shock, oxidative stress, DNA damage or cytotoxic drugs, the permeability of the inner mitochondrial membrane increases, and the mitochondrial membrane potential is disrupted (21). Subsequently, cytochrome $c$ is released, triggering caspase-9, and downstream effectors caspase-3 and/or -7 activation (21).

To elucidate the mechanism of apoptosis elicited by baicalin in HL-60 cells, the protein expression of caspase-3, -8 , and -9 was detected using western blotting. Consistent with previous reports $(6,8)$, the results of the present study demonstrated that caspase-3 and -9 were cleaved, and activated following baicalin treatment, demonstrating a role for mitochondria in the process of ongoing apoptosis in HL-60 cells. Though caspase- 8 activation had been confirmed in cervical cancer HeLa cells (22) and in human colorectal carcinoma SW620 cells (23), the roles of caspase- 8 in baicalin-induced apoptosis in human leukemia HL-60 cells remained to be clarified. In the present study, increased expression of cleaved caspase- 8 was detected following exposure to baicalin for $12 \mathrm{~h}$, and more notably after $24 \mathrm{~h}$. Thus, it was postulated that, in addition to the intrinsic signaling pathway, the extrinsic pathway may also contribute to apoptosis induced by baicalin in HL-60 cells.

To further confirm the convergence in apoptosis signaling, molecular markers associated with the extrinsic and intrinsic pathways were analyzed. Similar to the results acquired from hepatoma cell lines (24) and the leukemia-derived $\mathrm{T}$ cell line (6), it was demonstrated that baicalin caused $\Delta \Psi \mathrm{m}$ disruption of HL-60 cells and increased the expression of Bax, while it decreased expression of $\mathrm{Bcl}-2$, confirming that the intrinsic pathway participated in apoptosis induced by baicalin.

It is well established that caspase- 8 activation is initiated by the activation of death receptors. Currently, six distinct death receptors are known, including Fas (APO-1/CD95), the TRAIL receptor (R)-1 (also named DR4), TRAIL-R2 (also named DR5), tumor necrosis factor-R1, DR3 and DR6. Of these receptors, Fas and the TRAIL receptors have been extensively investigated (25). A previous study validated that modulation of Fas expression on the surface of tumor cells may potentiate the induction of apoptosis in tumor cells in response to chemo- and immuno-therapeutic agents (26). In addition, baicalin has been reported to trigger Fas and FasL expression during HeLa cell apoptosis (21). However, in the T-cell acute lymphoblastic leukemia cell line CCRF-CEM, it appeared that baicalin treatment exhibited no significant effect on the expression of Fas (7). In the present study, the upregulation of Fas and its ligand FasL was detected at the mRNA, and protein level. However, there was no change in DR4 and DR5 mRNA expression. This discrepancy is likely attributed to the specific cell type investigated. The data of the present study implied that the Fas receptor, but not DR4 and DR5, was involved in baicalin-induced caspase- 8 activation.

An investigation regarding the involvement of transcription factors in cell proliferation may be useful to perform a more in-depth analysis of the exact mechanism by which baicalin exhibits antitumor activity. c-Myc, as a transcription factor, serves an important role in cell proliferation and is overexpressed in numerous human tumors (27). Evidence has demonstrated that c-Myc binds to thousands of promoters of its target genes, including hTERT (28). It has been confirmed that c-Myc is involved in the control of telomerase activity, which is associated with cell proliferation in normal cells and tumors (29), through its ability to induce the transcriptional activation of hTERT. In the present study, the downregulation of c-Myc was detected at the mRNA and protein level, in addition, transcriptional activation of hTERT was also decreased, in line with the inhibition of HL-60 cells.

In conclusion, the results of the current study demonstrated that baicalin is able inhibit the proliferation of the AML HL60 cell line through $\mathrm{G}_{0} / \mathrm{G}_{1}$ phase arrest and significant apoptosis induction via the intrinsic, and extrinsic pathways. In addition, the inhibition of HL-60 cell growth was also mediated by telomerase inhibition through suppression of c-Myc. These results raise the possibility that baicalin may be a promising regimen for treatment of AML.

\section{Acknowledgements}

The authors would like to thank Dr Xiao Wang (Shandong Analysis and Test Center, Shandong Academy of Sciences) for providing purified baicalin. The present study was supported by the National Natural Science Foundation of China (grant nos. 8117292 and 81573467), the 'Twelfth Five-Year' National Science and Technology Support Program (grant no. 2013BAI07B02), the Natural Science Foundation 
of Shandong Province of China (grant nos. ZR2011HL045, ZR2015YL028, ZR2014HM085 and 2015ZRC03102), the Key Science and Technology Program of Shandong (grant no. 2013YD18031), the Project for Laureate of Taishan Scholar (grant no. ts201511075) and The Innovation Project of Shandong Academy of Medical Science.

\section{References}

1. Huang WH, Lee AR and Yang $\mathrm{CH}$ : Antioxidative and anti-inflammatory activities of polyhydroxyflavonoids of Scutellaria baicalensis GEORGI. Biosci Biotechnol Biochem 70: 2371-2380, 2006.

2. Shang X, He X, He X, Li M, Zhang R, Fan P, Zhang Q and Jia Z: The genus Scutellaria an ethnopharmacological and phytochemical review. J Ethnopharmacol 128: 279-313, 2010.

3. Ikezoe T, Chen SS, Heber D, Taguchi H and Koeffler HP: Baicalin is a major component of PC-SPES which inhibits the proliferation of human cancer cells via apoptosis and cell cycle arrest. Prostate 49: 285-292, 2001.

4. Zheng J, Hu JD, Chen YY, Chen BY, Huang Y, Zheng ZH and Liu TB: Baicalin induces apoptosis in leukemia HL-60/ADR cells via possible down-regulation of the PI3K/Akt signaling pathway. Asian Pac J Cancer Prev 13: 1119-1124, 2012.

5. Parajuli P, Joshee N, Rimando AM, Mittal S and Yadav AK: In vitro antitumor mechanisms of various Scutellaria extracts and constituent flavonoids. Planta Med 275: 41-48, 2009.

6. Ueda S, Nakamura H, Masutani H, Sasada T, Takabayashi A, Yamaoka Y and Yodoi J: Baicalin induces apoptosis via mitochondrial pathway as prooxidant. Mol Immunol 38: 781-791, 2002.

7. Shieh DE, Cheng HY, Yen MH, Chiang LC and Lin CC: Baicalin-induced apoptosis is mediated by Bcl-2-dependent, but not p53-dependent, pathway in human leukemia cell lines. Am J Chin Med 34: 245-261, 2006.

8. Lu HF, Hsueh SC, Ho YT, Kao MC, Yang JS, Chiu TH, Huamg SY, Lin CC and Chung JG: ROS mediates baicalin-induced apoptosis in human promyelocytic leukemia HL-60 cells through the expression of the Gadd153 and mitochondrial-depedent pathway. Anticancer Res 27: 117-125, 2007.

9. Grandori C, Cowley SM, James LP and Eisenman RN: The $\mathrm{Myc} / \mathrm{Max} / \mathrm{Mad}$ network and the transcriptional control of cell behavior. Annu Rev Cell Dev Biol 16: 653-699, 2000.

10. Felsher DW: MYC inactivation elicits oncogene addiction through both tumor cell-intrinsic and host-dependent mechanisms. Genes Cancer 1: 597-604, 2010.

11. Sirinian MI, Pisegna S, Paroli M, Militi S, Testa U and Peschle C: Zinc modulates c-Myc/Mad1 balance in human leukemia cells. Leukemia 17: 272-274, 2003.

12. Huang ST, Wang CY, Yang RC, Chu CJ, Wu HT and Pang JH: Wogonin, an active compound in Scutellaria baicalensis, induces apoptosis and reduces telomerase activity in the HL-60 leukemia cells. Phytomedicine 17: 47-54, 2010.
13. Ren X, Zhang Y, Li C, Wang H, Jiang Z, Zhang Z, Guo Q, Song G, Bi K and Jiang G: Enhancement of baicalin by hexamethylene bisacetamide on the induction of apoptosis contributes to simultaneous activation of the intrinsic and extrinsic apoptotic pathways in human leukemia cells. Oncol Rep 30: 2071-2080, 2013.

14. Kyo S, Takakura M, Taira T, Kanaya T, Itoh H, Yutsudo M, Ariga $\mathrm{H}$ and Inoue M: Sp1 cooperates with c-Myc to activate transcription of the human telomerase reverse transcriptase gene (hTERT). Nucleic Acids Res 28: 669-677, 2000.

15. Riedl SJ and Shi Y: Molecular mechanisms of caspase regulation during apoptosis. Nat Rev Mol Cell Biol 5: 897-907, 2004.

16. Gottlieb E, Armour SM, Harris MH and Thompson CB: Mitochondrial membrane potential regulates matrix configuration and cytochrome c release during apoptosis. Cell Death Differ 10: 709-717, 2003.

17. Ghavami S, Hashemi M, Ande SR, Yeganeh B, Xiao W, Eshraghi M, Bus CJ, Kadkhoda K, Wiechec E, Halayko AJ and Los M: Apoptosis and cancer: Mutations within caspase genes. J Med Genet 46: 497-510, 2009.

18. Steinman RA: Cell cycle regulators and hematopoiesis. Oncogene 21: 3403-3413, 2002.

19. Lavrik IN, Golks A and Krammer PH: Caspases: Pharmacological manipulation of cell death. J Clin Invest 115: 2665-2672, 2005.

20. Fulda S: Caspase 8 in cancer biology and therapy. Cancer Lett 281: 128-133, 2009.

21. Arnoult D: Mitochondrial fragmentation in apoptosis. Trends Cell Biol 17: 6-11, 2007.

22. Peng Y, Fu ZZ, Guo CS, Zhang YX, Di Y, Jiang B and Li QW: Effects and mechanism of baicalin on apoptosis of cervical cancer HeLa cells In-vitro. Iran J Pharm Res 14: 251-261, 2015.

23. Chen WC, Kuo TH, Tzeng YS and Tsai YC: Baicalin induces apoptosis in SW620 human colorectal carcinoma cells in vitro and suppresses tumor growth in vivo. Molecule 17: 3844-3857, 2012.

24. Chang WH, Chen $\mathrm{CH}$ and Lu FJ: Different effects of baicalein, baicalin and wogonin on mitochondrial function, glutathione content and cell cycle progression in human hepatoma cell lines. Planta Med 68: 128-132, 2002.

25. Thorburn A: Death receptor-induced cell killing. Cell Signal 16: $139-144,2004$

26. Kolenko VM, Uzzo RG, Bukowski R and Finke JH: Caspase-dependent and -independent death pathways in cancer therapy. Apoptosis 5: 17-20, 2000.

27. Meyer N and Penn LZ: Reflecting on 25 years with MYC. Nat Rev Cancer 8: 976-990, 2008.

28. Nie Z, Hu G, Wei G, Cui K, Yamane A, Resch W, Wang R, Green DR, Tessarollo L, Casellas R, et al: c-Myc is a universal amplifier of expressed genes in lymphocytes and embryonic stem cells. Cell 151: 68-79, 2012

29. Biroccio A, Amodei S, Benassi B, Scarsella M, Cianciulli A, Mottolese M, Del Bufalo D, Leonetti C and Zupi G: Reconstitution of hTERT restores tumorigenicity in melanoma-derived c-Myc low-expressing clones. Oncogene 21: 3011-3019, 2002. 